\title{
The whereabouts of visual attention: Involuntary attentional bias toward the default gaze direction
}

\author{
Ryoichi Nakashima $^{1,2} \cdot$ Takatsune Kumada ${ }^{1,3}$
}

Published online: 12 May 2017

(C) The Psychonomic Society, Inc. 2017

\begin{abstract}
This study proposed and verified a new hypothesis on the relationship between gaze direction and visual attention: attentional bias by default gaze direction based on eyehead coordination. We conducted a target identification task in which visual stimuli appeared briefly to the left and right of a fixation cross. In Experiment 1, the direction of the participant's head (aligned with the body) was manipulated to the left, front, or right relative to a central fixation point. In Experiment 2, head direction was manipulated to the left, front, or right relative to the body direction. This manipulation was based on results showing that bias of eye position distribution was highly correlated with head direction. In both experiments, accuracy was greater when the target appeared at a position where the eyes would potentially be directed. Consequently, eye-head coordination influences visual attention. That is, attention can be automatically biased toward the location where the eyes tend to be directed.
\end{abstract}

Keywords Premotor theory of attention - Gaze direction . Head direction · Visual attention · Eye-head coordination

To perceive visual environments, people usually focus visual attention on one object and/or location at one time, and shift attention sequentially. A visual attentional shift usually correlates with eye movement. The relationship between attention

Ryoichi Nakashima

nakashima.ryo1@gmail.com

1 RIKEN BSI-TOYOTA Collaboration Center, RIKEN2-1 Hirosawa, Wako, Saitama 351-0198, Japan

2 The University of Tokyo, Tokyo, Japan

3 Kyoto University, Kyoto, Japan and eye movement has been widely investigated; for example, it is found in saccadic eye movement (e.g., Hoffman \& Subramaniam, 1995), smooth pursuit eye movement (e.g., Lovejoy, Fowler, \& Krauzlis, 2009), vergence eye movement (Erkelens \& Collewijn, 1991), and eye movements during blinks (Irwin, 2011). The former three examples involve voluntary eye movements, and the latter is an involuntary eye movement.

The premotor theory of attention was proposed and developed based on numerous results showing a correlation between attention and eye movement, especially saccadic eye movement (e.g., Rizzolatti, Riggio, Dascola, \& Umiltá, 1987; Smith \& Schenk, 2012). This theory suggests that shifts of visual attention are accomplished by preparing an eye movement program that executes a saccade to the attended location via activity in oculomotor areas in the brain (e.g., the superior colliculus, the frontal eye field). Furthermore, recent studies have indicated that an attentional shift can be produced even by an eye movement program without intention; blinking (Irwin, 2011), or reflexive saccade (Peterson, Kramer, \& Irwin, 2004). Thus, irrespective of the intention of an eye movement, visual attention moves to the target location of the eye movement before the eye movement is actually executed. This theory also aligns with the wellestablished notion that attention is usually directed on or around a gaze position (cf. Henderson, 2003).

We recently demonstrated that perception of a visual target is modulated by the observer's head direction (Nakashima \& Shioiri, 2014, 2015). Specifically, in our previous experiment (Nakashima \& Shioiri, 2015), participants identified a target presented far from the fixation cross (i.e., in their peripheral vision). The critical manipulation was their head direction: toward the fixation cross, toward the target location, or toward a location completely different from the former two. When the observer's head was directed toward the target location, target 
identification was facilitated, relative to when the head was directed toward locations other than the target. The results indicated that attention is biased by head direction; this is inconsistent with the conventional suggestion that attention is usually located around a gaze position.

Our recent studies have shown that head direction, as well as gaze position, influences visual attention. However, it remains unclear why this is so. To clarify the basis for this finding, this study proposes a new hypothesis about the relationship between gaze direction and visual attention, namely, attentional bias by default gaze direction. In this context, default gaze direction is defined as the most spontaneous gaze direction based on an observer's posture when no eye movement is intended. Through this hypothesis, we argue that there is a default gaze direction relative to head direction based on eye-head coordination (e.g., head front direction), and attention can be involuntarily biased toward the default gaze direction independently of actual and/or intended eye movement. This hypothesis can explain results that have shown facilitation of visual perception by head direction. When posture is natural, with head and body aligned, eye movements during eccentric gaze (i.e., the gaze direction is misaligned from the head direction) are faster and shorter in latency toward a central head position than toward the periphery (side) of the head (e.g., Fuller, 1996; Krebs, Schoenfeld Beohler, Song, \& Woldorff). In addition, people usually look at objects straight ahead (cf. the central fixation bias; Tatler, 2007). Thus, eyes should potentially be directed at a position that aligns with the center of the head. Following this, visual attention should be also biased toward a location in front of the observer's head.

The goal of the present study was to verify the hypothesis that visual attention is biased by default gaze direction. Two experiments manipulated head and body directions in order to clarify the relationship between visual attention and gaze direction (see Fig. 1). Experiment 1 (Experiments 1A and 1B) examined attentional bias when an observer's head and body were aligned and the direction of the head was manipulated: front, left, and right relative to a fixation location. That is, we compared performance between frontal and eccentric gazes. In addition, our recent studies reported that head direction relative to the body biases the gaze position distribution toward a location more in alignment with that of the head direction (Fang, Emoto, Nakashima, Matsumiya, Kuriki, \& Shioiri, 2015a; Fang, Nakashima, Matsumiya, Kuriki, \& Shioiri, 2015b; Nakashima, Fang, Hatori, Hiratani, Matsumiya, Kuriki, \& Shioiri, 2015). Based on those results, Experiment 2 (Experiments 2A and 2B) examined attentional bias when the head direction differed from the body direction; that is, the body direction was moved such that it was centrally aligned with the head direction, or it was shifted left or right relative to the head direction.

\section{Experiment 1A}

A number of studies have suggested that eyes may shift (involuntarily) to a central position of the head when people are in a natural posture where head and body are aligned (Fuller, 1996; Krebs et al., 2010). According to our hypothesis, attention is generally biased toward the central position when people look at an object with an eccentric gaze; this leads to facilitation of visual perception of the stimulus that is consistent with the head direction. For example, when visual stimuli that appear at a location to the left or right of a fixation location are looked at by a left eccentric gaze (i.e., eyes directed to the left from the head), the perception of the target stimulus at the right location should be better than that at the left location, even though the target location is not known.

\section{Method}

\section{Participants}

Eighteen volunteers (22-24 years old) participated in Experiment 1A. All of them had normal or corrected-tonormal vision and were naïve to the purpose of the study. All experiments were approved by the institutional review board of Riken, and written informed consent was obtained from all participants. The experiments in this study were conducted in accordance with the Declaration of Helsinki in the treatment of the observers.

\section{Stimuli}

The experimental display included two visual stimuli (a target and a distractor) presented $15^{\circ}$ to the left and right of the fixation (Fig. 1a). The target stimulus was a white T-shaped figure $\left(1^{\circ} \times 1^{\circ}\right.$ of visual angle at a viewing distance of $\left.60 \mathrm{~cm}\right)$, rotated $90^{\circ}$ to the left or right of vertical center (see Fig. 1a). The distractor stimulus was a white L-shaped figure $\left(1^{\circ} \times 1^{\circ}\right)$, rotated $0^{\circ}, 90^{\circ}, 180^{\circ}$, or $270^{\circ}$. The background display was uniform gray.

\section{Apparatus}

Stimuli were displayed on a 27 -inch display $(2560 \times 1440$ pixels). Stimulus presentation and response recording were controlled by a computer, using the MATLAB software and the Psychophysics Toolbox (Brainard, 1997; Pelli, 1997).

\section{Procedure}

Participants were seated in front of the display in a dark room. The viewing distance was $60 \mathrm{~cm}$. At the beginning of each trial, a fixation cross was presented at the center of the display. 
a

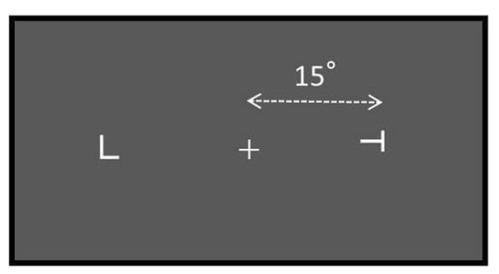

b

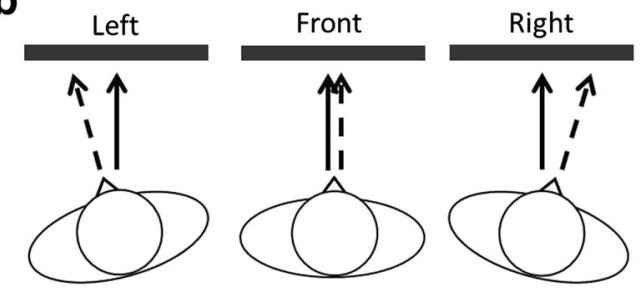

C

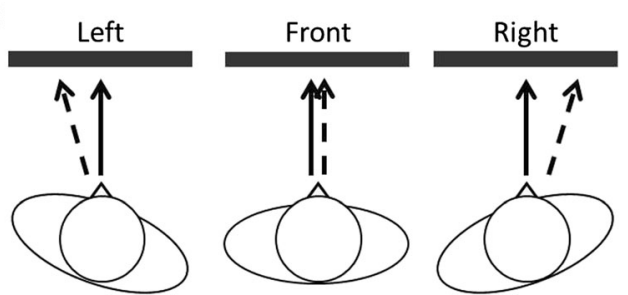

Fig. 1 a-c Experimental setups. a An example of a stimulus display where a target and distractor stimuli were presented at $15^{\circ}$ left and right from the fixation cross. $\mathbf{b}$ Head direction manipulation in Experiments $1 \mathrm{~A}$ and $1 \mathrm{~B}$ where a participant's head direction (aligned with the body direction) was front, left, or right relative to the fixation position. c Head direction manipulation in Experiments 2A and 2B where the participants' head direction was front, left, or right relative to the body direction. Solid lines Actual gaze direction, dashed lines direction where the eye potentially moves by eye-head coordination

Participants were told to gaze at the cross without moving their eyes from the cross throughout the trial. Participants pressed the start button to begin the trial, and after $500 \mathrm{~ms}$, two visual stimuli, one $15^{\circ}$ to the left and the other to the right of the cross, were presented for $100 \mathrm{~ms}$, followed by mask stimuli. The mask stimuli lasted until the response was made. The task was to identify the direction of the target (i.e., whether the bottom of the $\mathrm{T}$ was pointed to the right or left) as accurately as possible, and the accuracy of the response was measured. To minimize possible fatigue from holding an eccentric gaze, participants were not required to maintain gaze at the fixation cross between trials.

The participants' heads were fixed by a forehead and chin rest throughout an experimental block (Fig. 1b), and head direction (aligned with the body direction) was manipulated: front, left, or right. In the front condition, the head (and body) was directed to the center of the display, which was the location of the fixation cross; in the left (right) condition, the head (and body) was directed $15^{\circ}$ to the left (right) of the center of the display, i.e., the left (right) stimulus location.

The head direction conditions (front, left, right) were blocked, and block order was counterbalanced between participants. Each block included 120 trials: 60 trials under each of the two target position conditions (left, right). Trial order was randomized in each block. Before the experimental session, 20 practice trials were conducted to familiarize participants with the task in the front condition, and feedback on responses was given.

\section{Results and Discussion}

The percentages of correct responses as a function of head direction and target location are summarized in Fig. 2. A $3 \times$ 2 (head direction $\times$ target location) repeated measures analysis of variance (ANOVA) revealed that the interaction between head direction and target location was significant, $F(2,34)=$ 13.06, $P<.001, \eta_{\mathrm{p}}{ }^{2}=.43$, although the main effects were not significant, $F \mathrm{~s}<1$. Performance was higher in the left (or right) target location than in the opposite location in the left (or right) head direction: left head direction, $P<.001$; right head direction, $P=.003$. The level of accuracy did not differ significantly as function of target location for the frontal direction condition, $P=.57$.

We then compared the reaction times (RTs) on correct response trials in the head direction and target location conditions (see Table 1), although this was not our main purpose. An ANOVA revealed a significant interaction between head direction and target location, $F(2,34)=7.27, P=.002, \eta_{\mathrm{p}}{ }^{2}=.30$, with nonsignificant main effects, $F_{\mathrm{s}}<1$. RTs were shorter for the left (or right) target location than for the opposite location in the left (or right) head direction: left head direction, $P=.02$; right head direction, $P=.07$. RTs did not differ significantly as function of target location for the frontal direction, $P=.35$. These results were consistent with the results for accuracy, indicating that there was no speed-accuracy tradeoff.

In this experiment, since target location was randomized in a block and participants could not know the target position in each trial beforehand, the participants had no incentive to voluntarily direct attention to one of the locations. Nonetheless,

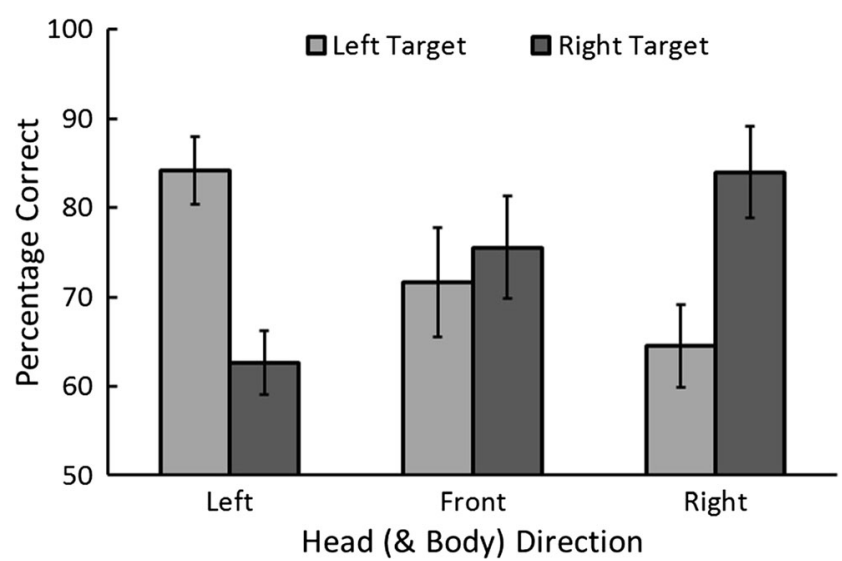

Fig. 2 Percentage of correct responses in Experiment 1A. Error bars $95 \%$ confidence intervals 
Table 1 Summary of reaction times $(\mathrm{msec})$ in the experiments $($ Mean $\pm S E)$

\begin{tabular}{llrrr}
\hline \multirow{2}{*}{ Target location } & \multicolumn{2}{l}{ Head direction } \\
\cline { 3 - 5 } & & \multicolumn{1}{l}{ Left } & \multicolumn{1}{l}{ Front } & \multicolumn{1}{c}{ Right } \\
\hline \multirow{2}{*}{ Exp1A } & Left & $653 \pm 41$ & $721 \pm 42$ & $760 \pm 45$ \\
& Right & $724 \pm 45$ & $737 \pm 45$ & $679 \pm 50$ \\
Exp1B & Left & $1028 \pm 71$ & $1046 \pm 58$ & $1075 \pm 80$ \\
& Right & $1103 \pm 76$ & $1009 \pm 65$ & $1025 \pm 56$ \\
Exp2A & Left & $727 \pm 53$ & $688 \pm 40$ & $677 \pm 38$ \\
& Right & $697 \pm 49$ & $710 \pm 50$ & $675 \pm 41$ \\
Exp2B & Left & $1086 \pm 93$ & $1039 \pm 73$ & $996 \pm 65$ \\
& Right & $1087 \pm 74$ & $992 \pm 71$ & $965 \pm 58$ \\
& & &
\end{tabular}

Note: Head direction indicates the head direction (aligned with the body direction) relative to the fixation position in Experiments 1A and 1B, and indicates the head direction relative to the body direction in Experiments $2 \mathrm{~A}$ and $2 \mathrm{~B}$

target identification was facilitated in the head direction, that is, the location where the eyes are potentially directed in a natural situation (cf. Tatler, 2007).

The facilitation of target identification at congruent head directions supports our hypothesis that visual attention can be biased toward the location where the eyes tend to be directed. Therefore, the suggestion that visual attentional process is influenced by head direction (Nakashima \& Shioiri, 2014, 2015) can be explained by our present hypothesis of attentional bias by default gaze direction.

\section{Experiment 1B}

The results of Experiment 1A indicated that visual attention can be biased toward the location where the eyes tend to be directed. However, without recording gaze direction, we could not completely eliminate the possibility that participants incidentally directed their eyes to the target location in the head direction on some trials. If participants gazed at the target, the task would become very easy and performance would therefore be better. To examine this possibility, we conducted the same experimental task while recording participants' gaze direction.

\section{Method}

\section{Participants}

Twenty-four volunteers (20-24 years old) participated in Experiment 1B. All had normal or corrected-to-normal vision and were naïve to the purpose of the experiment.

\section{Stimuli and apparatus}

Stimuli and apparatus were the same as those in Experiment $1 \mathrm{~A}$ but with the additional use of a gaze recording system. Participants' gaze position data during trials were recorded by GazeParser (Sogo, 2013: http://gazeparser.sourceforge. net/index.html) and a motion capture camera (Optitracks v10r2, NaturalPoint, Corvallis, OR ; $640 \times 480$ pixels, $100 \mathrm{~Hz})$

\section{Procedure}

The sequence of each trial was the same as that in Experiment $1 \mathrm{~A}$, but to reduce participants' burden and fatigue, the number of trials was decreased to 80 (40 trials in each target location condition). An additional difference was that we recorded participants' gaze direction during each trial. After the instructions and the practice session, three experimental sessions were conducted. At the beginning of each experimental session, the experimenter calibrated the eyetracker by requiring participants to fixate nine markers sequentially. Calibration was considered accurate if the computer's estimate of the fixation position was within 50 pixels of each marker on average. The eyetracker recorded the position of the participant's left eye. The order of the three head direction conditions (i.e., experimental sessions) was randomized between participants.

\section{Results and Discussion}

It should be noted that gaze tracking was very difficult, especially during eccentric gazes, because the pupil was often occluded by an eyelid and not adequately recorded by the camera. As a result, gaze recording data were collected from only 12 of the 24 participants. Before the analysis, from the data of 12 gaze-recorded participants, we omitted trials on which gaze was not recorded at all $(2.4 \%$ of the trials were removed). We also omitted trials on which mean gaze position was more than $3^{\circ}$ away from the fixation location for more than $300 \mathrm{~ms}$ ( $6.4 \%$ of the trials were removed). To improve statistical power, we analyzed the performance accuracy of all participants, adding the data of the all trials of participants whose gaze recording data were not collected (i.e., gazeunrecorded participants) to the data described above.

Figure 3 shows the percentages of correct responses in Experiment 1B, collapsed over the gaze-recorded and gazeunrecorded groups because there were no significant interactions between group and the other conditions. We conducted an ANOVA with group as the between-participants factor and head direction and target location as within-participants factors. The main effect of group did not reach significance, $F(1$, 22) $=2.51, P=.13, \eta_{\mathrm{p}}{ }^{2}=.10$. Based on this, we assumed that the two groups were equivalent. We believe that all participants did not intentionally violate the instructions by looking 


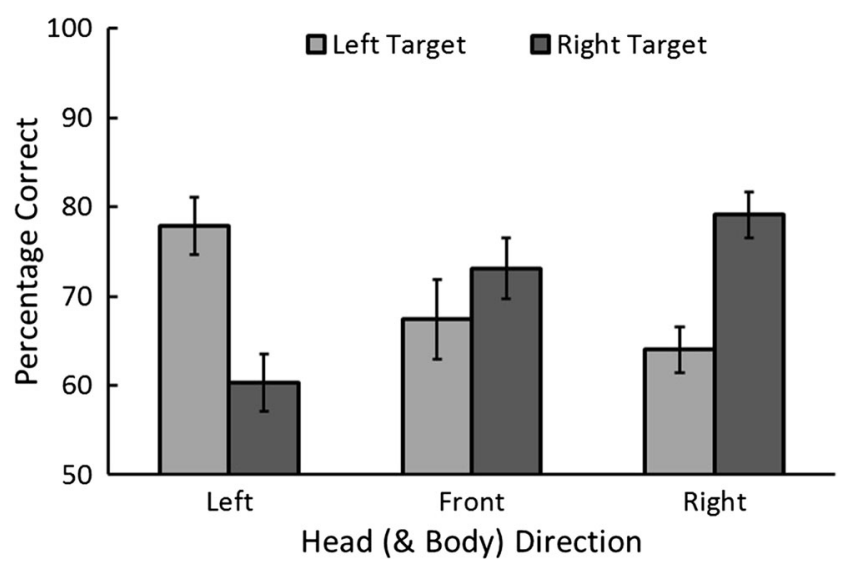

Fig. 3 Percentages of correct responses in Experiment 1B $(N=24)$. Error bars $95 \%$ confidence intervals

at the target position, because they were aware that their gaze position was being recorded.

Importantly, only the interaction between head direction and target location was significant, $F(2,44)=21.64$, $P<.001, \eta_{\mathrm{p}}{ }^{2}=.50$; again, the main effects and the other interactions were not significant, $F_{\mathrm{S}}<1$. Similar to the results in Experiment 1A, performance was higher in the left (right) target location than in the opposite location in the left (right) head direction, $P \mathrm{~s}<.001$. The level of accuracy did not differ significantly as function of target location for the frontal direction, $P=.18$.

We then conducted an ANOVA on RT data (see Table 1). The interaction between head direction and target location was marginally significant, $F(2,44)=2.45, P=.09, \eta_{\mathrm{p}}{ }^{2}=.10$. In the left head direction, RTs were shorter for the left target location than for the right target location, $P=.06$. The differences between the other conditions were not significant, $P \mathrm{~s}>$ .29. These results were partially consistent with the results for accuracy, indicating at least that there was no speed-accuracy tradeoff.

It should be noted that performance was better overall in Experiment 1A (without gaze recording, $N=18$ ) than in Experiment 1B (with gaze recording, $N=12$ ) for both accuracy, $F(1,28)=5.06, P=.03, \eta_{\mathrm{p}}{ }^{2}=.15$, and RT, $F(1,28)=$ $16.06, P<.001, \eta_{\mathrm{p}}{ }^{2}=.36$. There were no interactions of experiment and other conditions, $F \mathrm{~s}<1.3, P \mathrm{~s}>.27$. Although participants may have gazed at the target location accidentally on some trials, this did not influence the effect of head direction on visual perception.

We obtained results similar to those of Experiment 1A, even when gaze locations were recorded and trials were discarded on which gaze location was away from the fixation cross. The results strongly suggest that target identification was facilitated at congruent head directions. This supports our hypothesis that visual attention can be biased toward the location where the eyes tend to be directed.

\section{Experiment 2A}

Previous studies on body movement control reported that movements of eyes and head are coordinated to look at an object that appears at the periphery (e.g., Freedman, 2008; Oommen \& Stahl, 2005; Stahl, 1999). When people look at an object, both their eyes and head usually move toward the object. In addition to the eye-head movement coordination during a single gaze shift, our recent studies reported that static head direction relative to the body influences the distribution of gaze positions (Fang, Emoto et al., 2015, Fang, Nakashima et al., 2015; Nakashima et al., 2015). Specifically, when the head directs to the left (or right) relative to a particular body direction, the eye direction distribution also shifts to the left (or right) relative to the head. Thus, eyes should potentially direct to the left (or right) relative to the head when the head is directed to the left (or right) relative to the body. Combining these results and our hypothesis, it is possible that attention can be biased toward the left (or right) relative to the head when the head is directed toward the left (or right) relative to the body, leading to the facilitation of visual perception of a stimulus at this direction.

\section{Method}

\section{Participants, stimuli, apparatus, and procedure}

Eighteen young adults (22-24 years old) participated in Experiment 2A. Visual stimuli and apparatus were the same as those of Experiment 1. The experimental procedure was the same as that of Experiment 1A, except for the head direction manipulation. In Experiment 2A, the head and body directions were misaligned in the left/right head direction condition (Fig. 1c). The head direction was the same as the body direction in the front condition, and was left (right) relative to the body in the left (right) condition. Actually, the absolute head direction was always to the center of the display, and only body direction was manipulated. Body direction was fixed with stiff cushions in a way intended to cause no discomfort. The degree of misalignment between head and body was about $15-20^{\circ}$.

\section{Results and Discussion}

The percentages of correct responses as a function of head direction and target location are summarized in Fig. 4. An ANOVA revealed a significant interaction between head direction and target location, $F(2,34)=6.17, P=.005, \eta_{\mathrm{p}}{ }^{2}=.27$, although the main effects were not significant, $F \mathrm{~s}<1$. Performance was higher in the left (or right) target location than in the opposite location in the left (or right) head direction relative to the body condition: left head direction, $P=.02$; right head direction, $P=.04$. Accuracy levels were not 


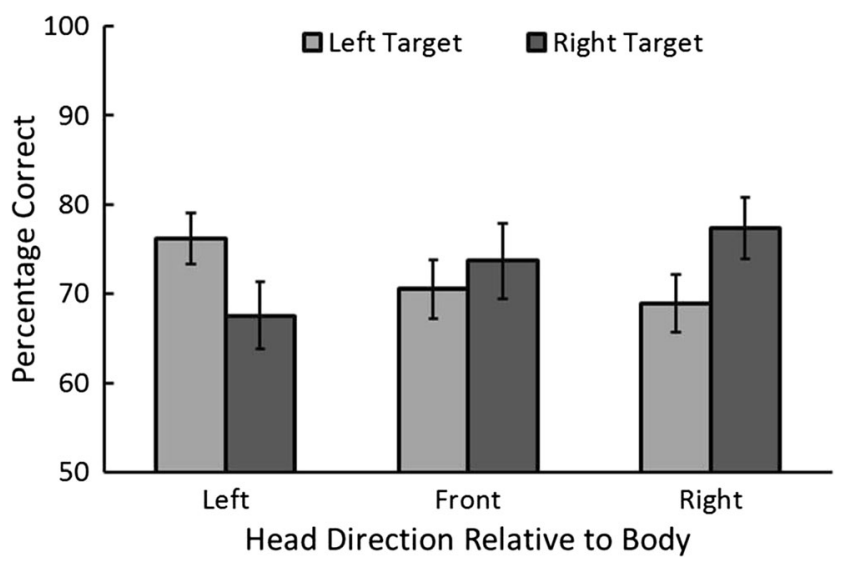

Fig. 4 Percentages of correct responses in Experiment 2A. Error bars $95 \%$ confidence intervals

significantly different between the target locations in the front head direction, $P=.5$.

An ANOVA on RT (see Table 1) revealed a marginally significant interaction between head direction and target location, $F(2,34)=3.21, P=.053, \eta_{\mathrm{p}}{ }^{2}=.16$, with no significant main effects, $F_{\mathrm{s}}<1$. Despite the interaction, there were no significant differences between any two conditions, $P \mathrm{~s}>.3$. Thus, there was once again no speed-accuracy tradeoff.

Although participants always gazed straight ahead at the fixation cross in this experiment, this could have been an unnatural position for the eyes relative to the head in some conditions. A supposition about static eye-head coordination (Fang, Emoto et al., 2015, Fang, Nakashima et al., 2015; Nakashima et al., 2015) holds that the natural eye direction should be shifted toward the left (or right) relative to the head when the head is directed toward the left (or right) relative to the body. The present results indicate that visual perception of a target stimulus is facilitated when the target is presented at the location where the eyes tend to be directed via eye-head coordination. Thus, these results also support our hypothesis of attentional bias by default gaze direction.

\section{Experiment 2B}

The purpose of Experiment 2B was to use eye gaze recording in conjunction with the task of Experiment 2A. We aimed to further confirm that attention is biased toward the location where the eyes are potentially directed (i.e., default gaze direction).

\section{Method}

\section{Participants}

Twenty-four volunteers (20-24 years old) participated in Experiment 2B. All had also participated in Experiment 1B, but those whose gaze data were recorded were not all the same as the corresponding participants in Experiment 1B.

Stimuli, apparatus and procedure

Stimuli, apparatus, and procedure were the same as those in Experiment 1B. The head direction manipulation was the same as that in Experiment 2A.

\section{Results and Discussion}

Gaze recording data were collected from only 13 of the 24 participants. Before the analysis, we omitted trials of these 13 participants on which gaze was not recorded at all (4.2\% of the trials were removed). Further, we omitted trials on which the mean gaze position was more than $3^{\circ}$ away from the fixation location for more than $300 \mathrm{~ms}$ ( $7.3 \%$ of the trials were removed). As in Experiment 1B, to improve the statistical power we analyzed the performance accuracy of all participants.

Figure 5 shows the percentages of correct responses in Experiment 2B collapsed over the gaze-recorded and gazeunrecorded groups, because there were no interactions of group and the other conditions. We conducted an ANOVA with group as the between-participants factor, and head direction and target location as within-participants factors. The main effect of group was marginally significant, $F(1,22)=$ $4.10, P=.055, \eta_{\mathrm{p}}{ }^{2}=.16$. Performance was higher in the gazeunrecorded tracking group than in the gaze-recorded group, because of individual differences and because of the inclusion of trials on which participants gazed at the target location accidentally.

Importantly, only the interaction between head direction and target location was significant, $F(2,44)=7.99$, $P=.001, \eta_{\mathrm{p}}{ }^{2}=.27$; the main effects and the other interactions were not significant, $F \mathrm{~s}<1$. As in Experiment $2 \mathrm{~A}$, performance was higher in the left (or right) target location than in the opposite location in the left (or right) head direction, $P \mathrm{~s}<$

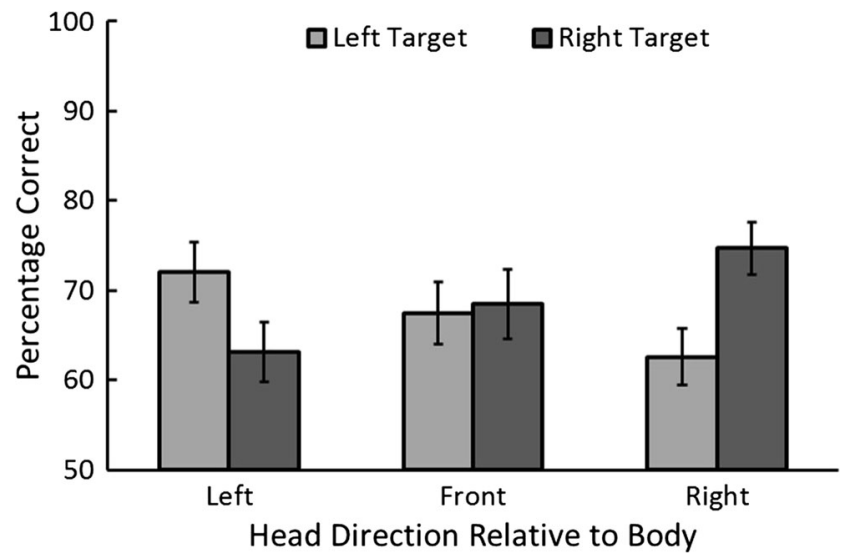

Fig. 5 Percentages of correct responses in Experiment 2B $(N=24)$. Error bars $95 \%$ confidence intervals 
.03 . The level of accuracy did not differ significantly as function of target location for the frontal direction, $P=.76$.

An ANOVA on RT data (see Table 1) revealed a marginally significant main effect of head direction, $F(2,44)=2.71, P=$ $.08, \eta_{\mathrm{p}}{ }^{2}=.11$, although noticeable differences were not obtained. These results, at least, suggest that there was no speedaccuracy tradeoff.

Overall performance was better in Experiment 2A (without gaze recording, $N=18$ ) than in Experiment $2 \mathrm{~B}$ (with gaze recording, $N=13)$, for both accuracy, $F(1,29)=5.77$, $P=.02, \eta_{\mathrm{p}}{ }^{2}=.17$, and RT, $F(1,29)=17.13, P<.001, \eta_{\mathrm{p}}{ }^{2}=$ .37. There were no significant interactions of experiment and the other conditions, $F_{\mathrm{S}}<1.7, P_{\mathrm{S}}>.19$. This indicates that participants in Experiment 2A gazed at target locations accidentally on some trials. We suggest, however, that the effect of head direction on visual perception was robust despite this accidental gazing.

We obtained results similar to those in Experiment 2A, even after discarding trials on which gaze was directed away from the fixation cross. Again, the results strongly suggest facilitation of target identification at congruent head directions, supporting our hypothesis that visual attention can be biased toward the location at which the eyes tend to be directed, based on static eye-head coordination (Fang, Emoto et al., 2015, Fang, Nakashima et al., 2015; Nakashima et al., 2015).

\section{General Discussion}

This study has proposed and verified a new hypothesis about the relationship between visual attention and gaze direction: attentional bias by default gaze direction. In Experiments $1 \mathrm{~A}$ and $1 \mathrm{~B}$, when the head and body were aligned, attention tended to be allocated to the center of the head. In Experiments $2 \mathrm{~A}$ and $2 \mathrm{~B}$, when the head and body were misaligned, attention tended to be allocated to the angle with greater deviation from the head direction. These results consistently indicate that attention is biased toward the location where the eyes are potentially directed (i.e., default gaze direction), and the visual perception of the stimulus is facilitated at this location. Thus, the eye-head coordination system affects the allocation of visual attention.

The premotor theory of attention (Rizzolatti et al., 1987; Smith \& Schenk, 2012) suggests that attentional shifts are accomplished by preparing an eye movement program. Unlike the premotor theory, which focuses on the relationship between dynamic eye movement and attention, we examined the relationship between static gaze direction and attention to suggest that visual attention is always biased by the default gaze position, that is, the gaze position based on the relationship between the directions of the head and body.

It is known that the eye movement system and the visual attention control system have a common neural basis (e.g., the superior colliculus, the frontal eye field). However, given the effect of head and body directions on attentional distribution via eye position, the scope of the neural computation may expand to include the states of the neck muscles and ocular muscles, and their relationship (cervico-ocular reflex; Barlow \& Freedman, 1980; Bronstein \& Hood, 1986). That is, attention may be biased by the signals of tension states of the ocular muscles. This takes into account the balance of ocular muscles in creating a stable gaze state without accompanying actual eye movements, in other words, independent of actual eye movements. Specifically, during an eccentric gaze, the balance of the ocular muscles is lost, and this generates a signal that is conveyed to the attention system. Signals from the neck muscles also influence ocular muscles; therefore head direction relative to the body determines the default gaze position as the stable gaze state. In this case, the state of the ocular muscles again conveys a signal to the attention system. Consequently, attention is biased automatically and involuntarily. The eye-head coordination (eye, head and body directions) influences attention via signals from ocular muscles.

The effect of eye-head coordination on visual attention, measured as the mean difference between performance at the default gaze location and that at the opposite location, was larger in Experiment $1 \mathrm{~A}(20.5 \%)$ than in Experiment $2 \mathrm{~A}$ $(8.5 \%), t(34)=2.34, P=.025$. More directly, when the same participants $(N=24)$ completed both tasks with gaze recording, similar results were obtained: Experiment 1B $(16.3 \%)$ and $2 \mathrm{~B}(10.5 \%), t(23)=2.08, P=.049$. During eccentric gaze, signals for the stable gaze state derive from the ocular muscles themselves. In contrast, when the head is directed laterally relative to the body, the signals conveyed are those from the neck muscles via the ocular muscles. Given that the ocular muscle control system and attention system have a common basis, the strength of the signals from the ocular muscle to the attention system should be larger when the origin of the signals is the ocular muscles themselves rather than the neck muscles. This disparity in signal strength can produce a difference in results.

We have shown that eye-head coordination influences visual attentional processing, thus introducing the idea that the location of the attentional focus can be estimated using information about the individual's head direction. Currently, head direction can be obtained easily from distant locations by various techniques, for example, by gait analysis (Nakazawa, Mitsugami, Yamazoe, \& Yagi, 2014), or through security cameras (Chamveha, Sugano, Sugimura, Siriteeralul, Okabe, Sato, \& Sugimoto, 2013). Thus, our findings can be applied to the estimation of attention in daily life, such as the attention of pedestrians, drivers, and many others involved in activities of motion.

In this study, we used GazeParser (Sogo, 2013), a relatively low-cost eye tracking system. To our knowledge, few experimental psychology studies have used this system to record 
participants' gaze locations (cf. Nakashima \& Kumada, 2017). Although we found this system to be useful, gaze recording was very difficult when the participant looked at a visual stimulus using eccentric gaze, and gaze data of about half of the participants could not be adequately recorded. Thus, in order to more effectively examine visual perception during eccentric gaze, it may be necessary to use other eye tracking systems or to employ other experimental methods, for example, including catch trials in which a target appears at a fixation location mixed in one block (cf. Nakashima \& Shioiri, 2015).

In conclusion, the present results suggest that visual attention is influenced by the eye-head coordination system and can be estimated based on head direction. We have demonstrated a new relationship between visual attention and gaze direction; attentional bias by default gaze direction. This suggestion expands the theory of visual attention.

Acknowledgments We thank Ryuta Iseki, Ritsuko Iwai, Sayako Ueda, and Kyoko Hine for helpful comments on our earlier manuscript. This study was supported by JSPS KAKENHI Grant Number 25780441 to R.N and TOYOTA Motor Corporation.

\section{References}

Barlow, D., \& Freedman, W. (1980). Cervico-ocular reflex in the normal adult. Acta Oto-Laryngologica, 89, 487-496.

Brainard, D. H. (1997). The psychophysics toolbox. Spatial Vision, 10, 443-446.

Bronstein, A. M., \& Hood, J. D. (1986). The cervico-ocular reflex in normal subjects and patients with absent vestibular function. Brain Research, 373, 399-408.

Chamveha, I., Sugano, Y., Sugimura, D., Siriteeralul, T., Okabe, T., Sato, Y., \& Sugimoto, A. (2013). Head direction estimation from low resolution images with scene adaptation. Computer Vision and Image Understanding, 117, 1502-1511.

Erkelens, C. J., \& Collewijn, H. (1991). Control of vergence: Gating among disparity inputs by voluntary target selection. Experimental Brain Research, 87, 671-678.

Fang, Y., Emoto, M., Nakashima, R., Matsumiya, K., Kuriki, I., \& Shioiri, S. (2015). Eye-position distribution depending on head orientation when observing movies on ultrahigh-definition television. ITE Transactions on Media Technology and Applications, 3, 149154.

Fang, Y., Nakashima, R., Matsumiya, K., Kuriki, I., \& Shioiri, S. (2015). Eye-head coordination for visual cognitive processing. PLOS ONE, 10(3), e0121035. doi:10.1371/journal.pone.0121035

Freedman, E. G. (2008). Coordination of the eyes and head during visual orienting. Experimental Brain Research, 190, 369-387.
Fuller, J. H. (1996). Eye position and target amplitude effects on human visual saccadic latencies. Experimental Brain Research, 109, 457466.

Henderson, J. M. (2003). Human gaze control during real-world scene perception. Trends in Cognitive Sciences, 7, 498-504.

Hoffman, J. E., \& Subramaniam, B. (1995). The role of visual attention in saccadic eye movements. Perception \& Psychophysics, 57, 787795.

Irwin, D. E. (2011). Where does attention go when you blink? Attention, Perception, \& Psychophysics, 73, 1374-1384.

Krebs, R. M., Schoenfeld, M. A., Boehler, C. N., Song, A. W., \& Woldorff, M. G. (2010). The saccadic re-centering bias is associated with activity changes in the human superior colliculus. Frontiers in Human Neuroscience, 4, 193. doi:10.3389/fnhum.2010.00193

Lovejoy, L. P., Fowler, G. A., \& Krauzlis, R. J. (2009). Spatial allocation of attention during smooth pursuit eye movements. Vision Research, 49, $1275-1285$.

Nakashima, R., \& Shioiri, S. (2014). Why do we move our head to look at an object in our peripheral region? Lateral viewing interferes with attentive search. PLoS ONE, 9(3), e92284. doi:10.1371/journal. pone.0092284

Nakashima, R., \& Shioiri, S. (2015). Facilitation of visual perception in head direction: Visual attention modulation based on head direction. PLoS ONE, 10(4), e0124367. doi:10.1371/journal.pone.0124367

Nakashima, R., \& Kumada, T. (2017). Peripersonal versus extrapersonal visual scene information for egocentric direction and position perception. The Quarterly Journal of Experimental Psychology. (in press). doi:10.1080/17470218.2017.1310267

Nakazawa, M., Mitsugami, I., Yamazoe, H., \& Yagi, Y. (2014). Head orientation estimation using gait observation. IPSJ Transactions on Computer Vision and Applications, 6, 63-67.

Nakashima, R., Fang, Y., Hatori, Y., Hiratani, A., Matsumiya, K., Kuriki, I., \& Shioiri, S. (2015). Saliency-based gaze prediction based on head direction. Vision Research, 117, 59-66.

Oommen, B. S., \& Stahl, J. S. (2005). Overlapping gaze shifts reveal timing of an eye-head gate. Experimental Brain Research, 167, 276-286.

Pelli, D. G. (1997). The VideoToolbox software for visual psychophysics: Transforming numbers into movies. Spatial Vision, 10, 437-442.

Peterson, M. S., Kramer, A. F., \& Irwin, D. E. (2004). Covert shifts of attention precede involuntary eye movements. Perception \& Psychophysics, 66, 398-405.

Rizzolatti, G., Riggio, L., Dascola, I., \& Umiltá, C. (1987). Reorienting attention across the vertical and horizontal meridians: Evidence in favor of a premotor theory of attention. Neuropsychologia, 25, 3140.

Smith, D. T., \& Schenk, T. (2012). The premotor theory of attention: Time to move one? Neuropsycholigia, 50, 1104-1114.

Sogo, H. (2013). GazeParser: An open-source and multiplatform library for low-cost eye tracking and analysis. Behavior Research Methods, 45, 684-695.

Stahl, J. S. (1999). Amplitude of human head movements associated with horizontal saccades. Experimental Brain Research, 126, 41-54.

Tatler, B. W. (2007). The central fixation bias in scene viewing: Selecting an optimal viewing position independently of motor biases and image feature distributions. Journal of Vision, 7(14), 4. 1-17. 